\title{
The efficacy and safety of silymarin in the treatment of chemotherapy-induced peripheral neuropathy, a randomized double-blinded clinical trial
}

\author{
Ramin Shekarriz ${ }^{1}$, Hojjat Ghorbani ${ }^{1}$, Mahmoud Mousazadeh $^{1}$, Khatereh Vahedi ${ }^{2}$, and \\ Ebrahim Salehifar ${ }^{3}$ \\ ${ }^{1}$ Mazandaran University of Medical Sciences \\ ${ }^{2}$ Isalmic Azad University \\ ${ }^{3}$ Mazandaran University of Medical Sciences Faculty of Pharmacy
}

August 7, 2021

\begin{abstract}
Aim: The aim of this study was to evaluate the efficacy and side effects of silymarin in the treatment of CIPN. Methods: Patients who referred to outpatient oncology department of a referral educational hospital affiliated to Mazandaran University of Medical Sciences and experienced CIPN were randomized to receive silymarin or placebo. Intervention group received 140 $\mathrm{mg}$ of the Silymarin twice daily accompanied and $300 \mathrm{mg} /$ day Gabapentin, whereas control group received $300 \mathrm{mg} /$ day of Gabapentin and placebo twice daily for 3 months. The grade of neuropathy was determined according to the CTCAE criterion. The improvement of neuropathy was defined as the reduction of at least one neuropathic score. The visual analogue scale (VAS) was used to assess the severity of patients' pain and the EORTC-QLQ-C30 criterion was used to assess the quality of life. Patients were evaluated initially and at the follow up visit 3 months after the enrollment. Results: A total of 80 patients were enrolled in the study. There was no significant difference between the groups in terms of severity of neuropathy at baseline. At the end of the study, the number of people with improved neuropathy in the silymarin group was $82.8 \%$ patients, which was significantly higher than $48.4 \%$ observed in the patients received placebo $(\mathrm{P}=0.005)$. The silymarin-treated group showed a significant reduction in pain compared with those receiving placebo. Despite the improvement in quality of life in the intervention group compared to the comparison group, this difference was not statistically significant. Gastrointestinal symptoms were the only reported side effects with a similar incidence in two groups. Conclusion: The present data demonstrate the potential clinical use of silymarin as an adjuvant therapy to reduce CIPN symptoms.
\end{abstract}

The efficacy and safety of silymarin in the treatment of chemotherapy-induced peripheral neuropathy, a randomized double-blinded clinical trial

Running Title: Silymarin in neuropathy of chemotherapy

\section{Abstract}

Aim: The aim of this study was to evaluate the efficacy and side effects of silymarin in the treatment of chemotherapy-induced peripheral neuropathy (CIPN).

Methods: Patients who referred to outpatient oncology department of a referral educational hospital affiliated to Mazandaran University of Medical Sciences and experienced CIPN were randomized to receive silymarin or placebo. Intervention group received $140 \mathrm{mg}$ of the Silymarin twice daily accompanied and 300 $\mathrm{mg}$ /day Gabapentin, whereas control group received $300 \mathrm{mg}$ /day of Gabapentin and placebo twice daily for 3 months. The grade of neuropathy was determined according to the CTCAE criterion. The improvement of 
neuropathy was defined as the reduction of at least one neuropathic score. The visual analogue scale (VAS) was used to assess the severity of patients' pain and the EORTC-QLQ-C30 criterion was used to assess the quality of life. Patients were evaluated initially and at the follow up visit 3 months after the enrollment.

Results: A total of 80 patients were enrolled in the study. There was no significant difference between the groups in terms of severity of neuropathy at baseline. At the end of the study, the number of people with improved neuropathy in the silymarin group was $82.8 \%$ patients, which was significantly higher than $48.4 \%$ observed in the patients received placebo $(\mathrm{P}=0.005)$. The silymarin-treated group showed a significant reduction in pain compared with those receiving placebo. Despite the improvement in quality of life in the intervention group compared to the comparison group, this difference was not statistically significant. Gastrointestinal symptoms were the only reported side effects with a similar incidence in two groups.

Conclusion: The present data demonstrate the potential clinical use of silymarin as an adjuvant therapy to reduce CIPN symptoms.

Keywords: Silymarin, Chemotherapy-induced peripheral neuropathy (CIPN), Inflammation, Platinum, Taxanes, Vinca alkaloids

\section{What is already known about this topic?}

- Chemotherapy-induces peripheral neuropathy is a common complication of chemotherapy agents with a significant impact on patients' quality of life.

- Oxidative stress and inflammation has a critical role in the development of chemotherapy-induces peripheral neuropathy.

- Silymarin was effective in reducing chemotherapy-induced neuropathic pain in animal studies. What does this article add?

- Silymarin significantly reduced the severity of chemotherapy-induces peripheral neuropathy as measured by Common Terminology Criteria for Adverse Events $(\mathrm{P}=0.005)$.

- The silymarin-treated group showed a significant reduction in pain compared with those receiving placebo $(\mathrm{P}=0.001)$.

- The frequency of adverse effects of silymarin was similar to placebo.

\section{Background}

Chemotherapy-induces peripheral neuropathy (CIPN) is a common complication of chemotherapy agents with a significant impact on patients' quality of life. The risk of developing CIPN is higher among conventional chemotherapy drugs especially with platinum (e.g., cisplatin and oxaliplatin), taxanes, and vinca alkaloids $[1,2]$.

The development of CIPN during antineoplastic therapy is one of the most common cause of the termination or modification of cancer treatment [3]. Peripheral neuropathy may present as sensory, motor, or autonomic dysfunction. Clinical signs of neurological complications include paresthesia, numbness of the fingers, gait disturbance, ataxia, loss of deep tendon reflexes, wrist or foot drop, facial nerve palsy, and optic neuropathy $[4,5]$.

The functional impairment of neurons induced by chemotherapeutic drugs may be mediated through oxidative stress, inflammation, apoptosis and electrophysiological disturbances [6]. In oxidative stress, the release of oxygen free radicals including hydrogen peroxide, superoxide ions, and nitric oxide causes changes in cell components which may lead to lipid peroxidation and subsequent cell death [7-9]. Silymarin is a flavonoid mixture extracted from Silybum marianum seed, which consists of a mixture of flavolignans including silibinin, isosilibinin, silicristin, and silidianin [10]. Although silymarin has been mostly known as its hepatoprotective properties [11], growing evidence has shown the neuroprotective effects of silymarin in neurodegenerative diseases, such as Alzheimer's disease, Parkinson's disease, and cerebral ischemia, via reducing reactive oxygen species (ROS), decreasing inflammatory cytokines, and altering cellular apoptosis pathway [12]. Beside the positive effect on CIPN, Laboratory data show that silymarin and its compounds 
(especially silibinin) have strong anticarcinogenic effect against cancer cells such as breast cancer and human promyelocytic leukemia HL-60 cells [13, 14].

It is acknowledged that the treatment of peripheral neuropathy is difficult and completely relieve of peripheral neuropathy (especially associated pain) may not occur with current therapies and complementary therapies may help in better management of CIPN $[4,15,16]$.

The aim of this study was to evaluate the efficacy and side effects of silymarin in the treatment of peripheral neuropathy caused by chemotherapy drugs.

\section{Methods}

\subsection{Patients:}

In our study, 236 patients who referred to the outpatient oncology department of Emam Hospital, affiliated to Mazandaran University of Medical Sciences were screened for enrollment. Eighty patients were eligible for the study. Inclusion criteria were a histologically confirmed diagnosis of cancer, at least 18 years of age, and occurrence of CIPN.

Exclusion criteria were 1) any underlying diseases with manifestation of neuropathy symptoms including diabetes, neuromuscular disease, vitamin B12 deficiency, amyloidosis, and connective tissue disease, 2) patients with a history of neurological diseases such as hereditary and acquired neuropathies, 3) taking any antioxidant supplement in the last two months, 4) pregnancy or breastfeeding, 4) known hypersensitivity to silymarin. All participants gave their written informed consent. Eligible patients were randomly assigned to treatment arms on a 1:1 basis.

The study was approved by the ethics committee of Mazandaran University of Medical Sciences (IR.MAZUMS.IMAMHOSPITAL.REC.1398.6176) and registered in Iranian Registry of Clinical Trials (IRCT20201128049515N1). The trial was conducted according to the guidelines of the Declaration of Helsinki for Research on Human Subjects 1989. Considering the effect size of 0.6, 95\% confidence level and test power of $80 \%$, sample size was calculated 80 patients (40 cases in each group) by G-Power software.

Figure 1: Here

\subsection{Study Design:}

This was a randomized, double-blind, placebo-controlled study. Patients treated with chemotherapy regimens consist of taxanes, platinum derivatives, vincristine or thalidomide and experienced symptoms of peripheral neuropathy were screened for enrollment. Block randomization with the size of four in each block was used to allocate patients in treatment or control arms. Each patient was assigned a computer-based unique fivedigit randomization code. The codes was held by a third party and handed over for statistical analyses after the complement of data collection. Both Silymarin and placebo were manufactured by an authorized pharmaceutical company (Goldaru, Isfahan, Iran). The placebo and silymarin were indistinguishable in size, weight, and their appearance. The treatment regimens was carried out according to the following scheme: Intervention group received $140 \mathrm{mg}$ of the silymarin in two daily doses with $300 \mathrm{mg} /$ day Gabapentin as a usual treatment of peripheral neuropathy, while the comparison group received $300 \mathrm{mg} /$ day of Gabapentin and placebo (filled by microcrystalline cellulose). The schedule of administration of placebo was equal to silymarin (twice daily). Duration of trial was 3 months. Patients were evaluated at baseline and 3 months after entering the study.

The primary objective was to evaluate the efficacy of the drug. Demographic and clinical characteristics of patients were recorded. Possible side effects of silymarin were recorded.

The CTCAE criterion (Common Terminology Criteria for Adverse Events) was used to assess the severity of neuropathy [17]. The grading of neuropathy was carried out based on the presence of paresthesia, peripheral motor neuropathy or peripheral sensory neuropathy and the level of limiting impact on activity of daily 
living (ADL) or self-care ADL. Improvement of neuropathy was defined as the reduction of at least one neuropathic score.

According to this criterion, a score of zero is equivalent to the absence of neuropathy, a score of 1 is equivalent to mild neuropathy, a score of 2 is equivalent to moderate neuropathy which limits instrumental ADL, a score of 3 is equivalent to severe neuropathy correspond which limits the self-care ADL, a score of 4 is equivalent to life-threatening neuropathy.

The visual analogue scale (VAS) was also used to assess the severity of patients' pain. The visual analogue scale was rated from 0 (no pain) to 10 (the most severe pain the patient has ever experienced). The EORTCQLQ-C30 (European Organization for Research and Treatment of Cancer - quality of life - questionnaires ) criterion was used to assess the improvement of patients' quality of life [18].

\subsection{Statistical Analysis:}

Mean and standard deviation were used to describe quantitative data and frequency and percentage were used to describe categorical data. ANCOVA test was used to evaluate the effect of silymarin on quantitative variables (EORTC QLQ and VAS) so that the final EORTC QLQ and VAS scores were used as the main factor and adjusted based on the initial score. Chi-square test was used to describe the qualitative outcomes (e.g., severity of neuropathy and number of patients improved). All tests were calculated at $95 \%$ significance level using SPSS software version 21.

\section{Results:}

In this trial, 236 patients were screened for eligibility and 80 patients enrolled (Figure 1).

The silymarin and placebo groups were similar in terms of sex, age and the frequency of each class of chemotherapy drugs used for patients (Table 1).

\section{Table 1: Here}

The mean score of EORTC QLQ of patients allocated to silymarin treatment was $94.07 \pm 6.65$, whereas it was $93.02 \pm 7.16$ in the placebo group. The mean final EORTC QLQ was $94.47 \pm 7.32$ in the intervention group and $92.42 \pm 8.39$ in the comparison group. In the present study, analysis of covariance showed that the mean differences of EORTC QLQ in the intervention group improved 1.06 times more than the comparison group, but this difference was not statistically significant $(\mathrm{p}$-value $=0.4)($ Table 2$)$.

\section{Table 2: Here}

The mean score of VAS in the Silymarin group and placebo group were $5.77 \pm 1.79$ and $5.73 \pm 1.98$, respectively. The mean of final VAS was $2.13 \pm 1.45$ in the silymarin group and $4.2 \pm 1.85$ in the placebo group. Analysis of covariance showed that VAS in the intervention group decreased 1.99 times (95\% CI: 1.001-2.975) more than the comparison group and this difference was statistically significant ( $\mathrm{p}$-value $<0.0001$ ) (Figure 2).

\section{Figure 2: Here}

The severity of CIPN was measured using the CTCAE criterion. There was no significant difference between the two groups in the severity of neuropathy at the beginning of the study (Table 3 ).

\section{Table 3: Here}

Improvement of neuropathy was defined as the reduction of at least one neuropathic score. The number of people with improved neuropathy was significantly higher in the silymarin group compared to placebo group (Table 4).

\section{Table 4: Here}

Six patients in placebo group and 7 patients in silymarin group experienced gastrointestinal symptoms (e.g., epigastric pain, nausea and vomiting). Three patients in silymarin group and 2 patients in placebo group 
discontinued the trial due to side effects. Overall, the side effects were tolerable and were managed with advising patients to receive the study drug after meal.

\section{Discussion}

To our knowledge, this is the first randomized, double-blind, placebo-controlled clinical trial to evaluate the efficacy and safety of silymarin in the treatment of CIPN. The silymarin-treated group showed a significant reduction in pain (measured by the VAS criterion) compared with those receiving placebo. Silymarin also significantly reduced the severity of peripheral neuropathy and more people in the silymarin-treated group showed improvement in peripheral neuropathy compared to the comparison group. But despite the improvement in quality of life in the intervention group compared to the comparison group, this difference was not statistically significant.

The development of CIPN during chemotherapy adversely affects the outcome of the disease and the patient's quality of life [19]. Cumulative doses of chemotherapy drugs, prior or concomitant administration of other neurotoxic drugs (such as cisplatin plus paclitaxel combination therapy), pre-existing neuropathy, co-morbidities and finally the short injection time of the drug are the most important risk factor for CIPN [2].

More than 40 randomized trials have evaluated a variety of pharmacologic interventions for the treatment of CIPN, only limited agents such as duloxetine and in some trials gabapentin were effective [20-22]. Due to the side effects of synthetic drugs following long-term use for painful and inflammatory conditions, many studies have tested different plant extracts and their active compounds for their analgesic and anti-inflammatory properties [23]. Oxidative stress and increased free radicals have been suggested as an important cause of CIPN. Silymarin, due to its antioxidant activity, may have protective effect against neuropathy [24]. Silymarin is a free radical scavenger that affects various stages of the arachidonic acid cascade via the cyclogenase and lipoxygenase pathways [7].

The present study showed significantly better improvement of CIPN following use of silymarin compared to placebo ( $82.8 \%$ vs. $48.4 \%$ ). The initial evidence of efficacy of silymarin in CIPN comes from experimental studies. Mannelli et al evaluated the efficacy of silybinin, an structural component of silymarin [25], in reducing oxaliplatin-induced neuropathic pain in a rat model. They reported that repeated administration of silybinin reduces oxaliplatin-induced pain. Silybinin was introduced as a valid treatment option for chemotherapy-induced neuropathy [26].

The effects of topical administration of silymarin in prevention of capecitabine-induced Hand-Foot Syndrome (HFS) were evaluated in a randomized, double-blinded, placebo controlled clinical trial. The trial concluded that prophylactic administration of topical silymarin could significantly reduce the severity of capecitabineinduced HFS and delay its occurrence in patients with gastrointestinal cancer after 9 weeks of application [27]. Choi et al. showed that oxaliplatin-induced neuropathy was inhibited by silymarin through combined mechanisms of combating oxidative stress, p38-mediated mitogen-activated protein kinase apoptosis, and decreased brain derived neurotrophic factor expression [28].

Following exposure to platinum agents, several morphological changes in DNA including damage to cell bodies, nuclei, nucleoli, neuronal atrophy in dorsal root ganglion cells and cell death may occur. In addition to platinum agents, ROS may have a role in the pathophysiology of CIPN following other chemotherapy agents such as taxane derivatives. Paclitaxel enhances the formation of ROS through alterations in mitochondria and subsequent pain caused by neurological and inflammatory damage through transient receptor potential ankyrin 1 (TRPA1) channels. It also leads to the production of Interleukin-1 $\beta$ (IL-1 $\beta$ ) and other proinflammatory cytokines secreted from microglia $[26,28]$. Vinca alkaloids, including vincristine cause inflammatory reactions in peripheral tissues and make changes in spinal afferent fiber. Increased $\mathrm{C}$ fiber degradation, calcium channel modulation, production of free radicals are mechanisms proposed for neuropathic pain following taxan derivatives. Also, they increase TNF- $\alpha$ and IL-6 in the sciatic nerve following oxidative stress $[29,30]$. Based on the aforementioned evidences, it seems that oxidative stress is the main cause of neuropathic pain by nearly all types of chemotherapy agents. So the beneficial effect of silymarin in CIPN could 
be contributed to its proven antioxidant properties [31].

Growing evidences suggest that different immune cells and subsequently inflammation are involved in the development of neuropathic pain. Activation of mast cells and secretion of inflammatory mediators (histamine and Tumor Necrosis Factor [TNF]- $\alpha$ ) may lead to peripheral nerve damage. Silymarin modulates the immune system by inhibiting neutrophil migration and immobilization of mast cells. It also inhibits TNF- $\alpha$ mediated production of reactive oxygen species, lipid peroxidation and modulates $\mathrm{T}$ cell function [32-34].

For an anti-neuropathic agent to be desirable in CIPN, it is necessary that the agent does not interfere with the cytotoxicity effect of the chemotherapy agent [35].

It was shown that silymarin poses a dual action as a chemopreventive agent and a chemosensitizer. Silymarin inhibits organic anion transporters (OAT) and ATP-binding cassettes (ABC) transporters which are helpful in overcoming the resistance to chemotherapy [36]. It may has a protective role against cancer in vivo and in vitro by inducing an imbalance between cell survival and apoptosis by disrupting the expression of cell cycle regulators and proteins involved in apoptosis [37]. Also anti-metastatic activity has been reported for silybinin [28].

Our study suffers from this limitation that it was not powered enough for subgroup analysis based on CIPN associated with different chemotherapeutic agents. Considering the positive findings of this trial, we recommend conducting future clinical trials to have a better understanding of usefulness of silymarin in treatment of CIPN induced by a given chemotherapeutic class.

\section{Conclusion:}

The results of this clinical trial demonstrate the potential clinical use of silymarin as an adjuvant therapy to reduce CIPN symptoms.

\section{Declarations}

\section{Ethics approval and consent to participate}

All procedures performed in study involving human participants were in accordance with the ethical standards of the institutional and/or national research committee and with the 1964 Helsinki declaration and its later amendments or comparable ethical standards. Also ethics committee of Mazandaran University of Medical Sciences, Iran approved this study (Code no: IR.MAZUMS.IMAMHOSPITAL.Rec.1398.6176).

\section{Consent for publication}

Not applicable

\section{Availability of data and materials}

The datasets used and/or analysed during the current study are available from the corresponding author on reasonable request.

\section{Competing interests}

The authors declare that they have no competing interests.

\section{Funding}

This study was funded by an academic grant from Mazandaran medical University.

\section{Authors' contributions}

R.Sh. and E.S. were involved in planning and supervised the work. H.Gh. and Kh.V. collected the data. M.M. performed the statistical analysis and aided in interpreting the results. R.Sh., H.Gh. and E.S. wrote the manuscript in consultation with M.M. All authors discussed the results and approved the final manuscript.

\section{Acknowledgements}


We would like to acknowledge the Research and Technology Deputy of Mazandaran University of Medical Sciences for financial support of the trial. Also we appreciate the Goldaru Company for free providing of the silymarin and placebo.

\section{Authors' information (optional)}

\section{List of abbreviations:}

ABC transporters: ATP-binding cassettes transporters

ADL: Activity of daily living

CIPN: Chemotherapy induced peripheral neuropathy

CTCAE: Common Terminology Criteria for Adverse Events

EORTC-QLQ-C30: European Organization for the Research and Treatment of Cancer Quality of Life Questionnaire

HFS: Hand-Foot Syndrome

IL: Interleukin

OAT: Organic anion transporters

ROS: Reactive oxygen species

TNF- $\alpha$ : Tumor necrosis factor-alpha

TRPA: Transient receptor potential ankyrin

VAS: Visual analog scale

\section{References:}

1. Wolf S, Barton D, Kottschade L et al. Chemotherapy-induced peripheral neuropathy: prevention and treatment strategies. European journal of cancer 2008; $44: 1507-15$.

2. Seretny M, Currie GL, Sena ES et al. Incidence, prevalence, and predictors of chemotherapy-induced peripheral neuropathy: a systematic review and meta-analysis. PAIN@ 2014; 155 : 2461-70.

3. Vitale MG, Barbato C, Crispo A et al. ZeOxaNMulti Trial: A Randomized, Double-Blinded, PlaceboControlled Trial of Oral PMA-Zeolite to Prevent Chemotherapy-Induced Side Effects, in Particular, Peripheral Neuropathy. Molecules 2020; 25 : 2297.

4. Quasthoff S, Hartung HP. Chemotherapy-induced peripheral neuropathy. Journal of neurology 2002; 249 : $9-17$.

5. Windebank AJ, Grisold W. Chemotherapy-induced neuropathy. Journal of the Peripheral Nervous System $2008 ; 13: 27-46$.

6. Gordon-Williams R, Farquhar-Smith P. Recent advances in understanding chemotherapy-induced peripheral neuropathy. F1000Research 2020;9 .

7. Surai PF. Silymarin as a natural antioxidant: an overview of the current evidence and perspectives. Antioxidants 2015; 4 : 204-47.

8. Areti A, Yerra VG, Naidu V, Kumar A. Oxidative stress and nerve damage: role in chemotherapy induced peripheral neuropathy. Redox biology 2014; 2 : 289-95.

9. Yowtak J, Lee KY, Kim HY et al. Reactive oxygen species contribute to neuropathic pain by reducing spinal GABA release. Pain(r) 2011;152: 844-52. 
10. Loguercio C, Festi D. Silybin and the liver: from basic research to clinical practice. World journal of gastroenterology: WJG 2011;17 : 2288.

11. Vargas-Mendoza N, Madrigal-Santillan E, Morales-Gonzalez A et al. Hepatoprotective effect of silymarin. World journal of hepatology2014; $6: 144$.

12. Borah A, Paul R, Choudhury S et al. Neuroprotective potential of silymarin against CNS disorders: insight into the pathways and molecular mechanisms of action. CNS neuroscience \& therapeutics 2013;19 : $847-53$.

13. Zi X, Feyes DK, Agarwal R. Anticarcinogenic effect of a flavonoid antioxidant, silymarin, in human breast cancer cells MDA-MB 468: induction of G1 arrest through an increase in Cip1/p21 concomitant with a decrease in kinase activity of cyclin-dependent kinases and associated cyclins. Clinical Cancer Research 1998; $4: 1055-64$.

14. Kang SN, Lee MH, Kim K-M et al. Induction of human promyelocytic leukemia HL-60 cell differentiation into monocytes by silibinin: involvement of protein kinase C. Biochemical pharmacology 2001;61 : 1487-95.

15. Chaudhry V, Rowinsky EK, Sartorius SE et al. Peripheral neuropathy from taxol and cisplatin combination chemotherapy: clinical and electrophysiological studies. Annals of Neurology: Official Journal of the American Neurological Association and the Child Neurology Society 1994; 35 : 304-11.

16. Ocean AJ, Vahdat LT. Chemotherapy-induced peripheral neuropathy: pathogenesis and emerging therapies. Supportive Care in Cancer 2004; 12 : 619-25.

17. Savarese D. Common terminology criteria for adverse events. Up ToDate Waltham, MA: UpToDate 2013.

18. Scott NW, Fayers P, Aaronson NK et al. EORTC QLQ-C30 reference values manual. 2008.

19. Hu S, Huang KM, Adams EJ et al. Recent developments of novel pharmacologic therapeutics for prevention of chemotherapy-induced peripheral neuropathy. Clinical Cancer Research 2019;25 : 6295-301.

20. Smith EML, Pang H, Cirrincione C et al. Effect of duloxetine on pain, function, and quality of life among patients with chemotherapy-induced painful peripheral neuropathy: a randomized clinical trial. Jama 2013; 309 : 1359-67.

21. Beijers A, Mols F, Vreugdenhil G. A systematic review on chronic oxaliplatin-induced peripheral neuropathy and the relation with oxaliplatin administration. Supportive Care in Cancer 2014;22 : 1999-2007.

22. Avan R, Janbabaei G, Hendouei $\mathrm{N}$ et al. The effect of pregabalin and duloxetine treatment on quality of life of breast cancer patients with taxane-induced sensory neuropathy: a randomized clinical trial.Journal of research in medical sciences: the official journal of Isfahan University of Medical Sciences 2018; 23.

23. Bahmani M, Shirzad H, Rafieian S, Rafieian-Kopaei M. Silybum marianum: beyond hepatoprotection. Journal of evidence-based complementary $\&$ alternative medicine 2015; 20 : 292-301.

24. Agarwal R, Agarwal C, Ichikawa $\mathrm{H}$ et al. Anticancer potential of silymarin: from bench to bed side. Anticancer research 2006;26 : 4457-98.

25. Valenzuela A, Garrido A. Biochemical bases of the pharmacological action of the flavonoid silymarin and of its structural isomer silibinin. Biological research 1994; 27 : 105-.

26. Mannelli LDC, Zanardelli M, Failli P, Ghelardini C. Oxaliplatin-induced neuropathy: oxidative stress as pathological mechanism. Protective effect of silibinin. The Journal of Pain2012; 13 : 276-84.

27. Elyasi S, Shojaee FSR, Allahyari A, Karimi G. Topical silymarin administration for prevention of capecitabine-induced hand-foot syndrome: A randomized, double-blinded, placebo-controlled clinical trial. Phytotherapy Research 2017; 31 : 1323-9. 
28. Choi J-Y, Yi HG, Park C-S et al. Inhibition of oxaliplatin-induced neurotoxicity by silymarin through increased expression of brain-derived neurotrophic factor and inhibition of p38-MAPK. Molecular $\&$ Cellular Toxicology 2019; $15:$ 145-52.

29. Soleimani V, Delghandi PS, Moallem SA, Karimi G. Safety and toxicity of silymarin, the major constituent of milk thistle extract: An updated review. Phytotherapy research 2019; 33 : 1627-38.

30. Campos R, Garrido A, Guerra R, Valenzuela A. Silybin dihemisuccinate protects against glutathione depletion and lipid peroxidation induced by acetaminophen on rat liver. Planta medica 1989; 55: 417-9.

31. Koksal E, GULCIN İ, Beyza S et al. In vitro antioxidant activity of silymarin. 2009.

32. D'Amico R, Impellizzeri D, Cuzzocrea S, Di Paola R. ALIAmides Update: Palmitoylethanolamide and its formulations on management of peripheral neuropathic pain. International Journal of Molecular Sciences 2020; $21: 5330$.

33. Tsuda M. Microglia-mediated regulation of neuropathic pain: molecular and cellular mechanisms. Biological and Pharmaceutical Bulletin 2019; 42 : 1959-68.

34. Inoue K, Tsuda M. Microglia in neuropathic pain: cellular and molecular mechanisms and therapeutic potential. Nature Reviews Neuroscience 2018; 19: 138.

35. Akbari Kordkheyli V, Nabipur E, Tafazoli A, Bagheri A. An overview on the effects of silibinin on different microRNAs expression in cancer.Journal of Mazandaran University of Medical Sciences 2018;28 : 213-29.

36. Delmas D, Xiao J, Vejux A, Aires V. Silymarin and cancer: A dual strategy in both in chemoprevention and chemosensitivity. Molecules 2020; $25: 2009$.

37. Jahanafrooz Z, Motamed N, Rinner B et al. Silibinin to improve cancer therapeutic, as an apoptotic inducer, autophagy modulator, cell cycle inhibitor, and microRNAs regulator. Life sciences 2018;213 : $236-47$.

\section{Hosted file}

Table 1.docx available at https://authorea.com/users/429582/articles/533264-the-efficacyand-safety-of-silymarin-in-the-treatment-of-chemotherapy-induced-peripheral-neuropathya-randomized-double-blinded-clinical-trial

\section{Hosted file}

Table 2.docx available at https://authorea.com/users/429582/articles/533264-the-efficacyand-safety-of-silymarin-in-the-treatment-of-chemotherapy-induced-peripheral-neuropathya-randomized-double-blinded-clinical-trial

\section{Hosted file}

Table 3.docx available at https://authorea.com/users/429582/articles/533264-the-efficacyand-safety-of-silymarin-in-the-treatment-of-chemotherapy-induced-peripheral-neuropathya-randomized-double-blinded-clinical-trial

\section{Hosted file}

Table 4.docx available at https://authorea.com/users/429582/articles/533264-the-efficacyand-safety-of-silymarin-in-the-treatment-of-chemotherapy-induced-peripheral-neuropathya-randomized-double-blinded-clinical-trial

\section{Hosted file}


Fig 1.docx available at https://authorea.com/users/429582/articles/533264-the-efficacy-andsafety-of-silymarin-in-the-treatment-of-chemotherapy-induced-peripheral-neuropathy-arandomized-double-blinded-clinical-trial

\section{Hosted file}

Fig 2.docx available at https://authorea.com/users/429582/articles/533264-the-efficacy-andsafety-of-silymarin-in-the-treatment-of-chemotherapy-induced-peripheral-neuropathy-arandomized-double-blinded-clinical-trial 